\title{
Towards a Personalized Cancer Gene Therapy: A Case of Clear Cell Renal Cell Carcinoma
}

\author{
Dumitru Andrei Iacobas ${ }^{*}$, Sanda Iacobas
}

Department of Pathology, New York Medical College, United States

Copyright $@ 2017$ by authors, all rights reserved. Authors agree that this article remains permanently open access under the terms of the Creative Commons Attribution License 4.0 International License

\begin{abstract}
An ideal cancer gene therapy would selectively destroy the cancer nodules with negligible effects on the surrounding not yet compromised tissue. This would be possible if the targeted genes are in command positions in the cancer but not in the normal cells. Logic dictates that, while being strongly protected by the homeostatic mechanisms, expression of a commander gene governs most major functional pathways by regulating the expression of numerous other genes. Owing to the cancer dependence on race, sex, age, genetic heritage, medical history, environmental and lifestyle associated risk factors each patient has most likely a distinct, dynamic and never repeatable set of commander genes. Here we introduce the "gene commanding height" as a measure of gene rank in the cell hierarchy and test our procedure on a surgically removed metastatic clear cell renal cell carcinoma. Results indicate that each histopathologically distinct region has a unique set of commander genes and that cancer cell commanders are in low positions in normal cells. We believe that the genomic oncology should identify the cancer cell commander genes of the individual patient instead of testing for the biomarkers selected from most frequently altered genes in large populations.
\end{abstract}

Keywords Commander Gene, Gene Commanding Height, Gene Networks, Genomic Fabric, Kidney Cancer

\section{Introduction}

Most cancers are caused by random changes in the genome of individual cells $[1,5]$. Tumors are not homogeneous but composed of several subpopulations of morphologically distinct cell types, pointing to different genetic alterations. Cancer development depends on race, sex, age, life-style, medical history, and environmental exposure to certain toxins and radiations [6]. A very important yet not well understood role in cancerization is played by the inhabiting microbiota (e.g. [7]) modulated by one's diet. However, most meta-analyses have not considered a well-stratified population with respect to all combinations of major risk factors. Instead, they collated data from several laboratories whose different platforms, protocols and data processing procedures question the significance (e.g. [8-13])

Numerous genomics labs are racing to identify the gene whose mutation, epigenetic alteration or/and regulation of the expression level indicate with reasonable predictive value the occurrence and development of a particular disease (e.g. $[14,15])$. Such "biomarkers" are selected from the most frequently altered genes in meta-analyses of large population cohorts. The millions of mutations in the genome of cancer cells [16] result in altered sequence and/or abundance of mRNA transcribed from thousands of affected genes. Although neglected and presumably not with equal effects, each of these alterations may contribute to the cancer phenotype. Experimental evaluation of the phenotypic effects of a particular gene alteration is almost impossible given that any gene manipulation affects hundreds of other genes as reported in countless studies on genetically engineered animals and cell lines (including ours, e.g. [17]). Owing to their astronomic number there is no way to consider and validate all possible combinations of genes whose mutation/regulation might be indicative for a particular form of cancer in each population category (e.g. there are $>17$ trillion distinct 10-gene panels selectable from 100 candidates!). Because of this, no bio-assay can, in principle, provide a reasonable predictive value in diagnosing a sporadic cancer [18-21]. There are essentially innumerable combinations of epigenetic alterations that regulate the transcriptome in spite of claims that a particular DNA hypermethylation is a pan-cancer marker [22].

Nonetheless, each cell should be governed by a set of commander genes whose right expression is critical for the cell phenotype, survival, proliferation or/and integration in a multicellular structure. As such, expression of a commander gene should regulate expression of numerous other genes while being highly protected by cell homeostasis. The strong protection makes a commander gene less alterable by the random alterations caused by the stochastic nature of the 
chemical reactions and therefore not selectable as a disease biomarker by the Principal Component Analysis [23]. However, when it happens, alteration of a commander gene reverberates through the entire cell transcriptome and is amplified by the alteration of the genes it commands. If the commander genes in cancer cells are commanded in normal ones then their smart manipulation might selectively destroy the cancer cells from a heterogeneous tumor and may have lesser effect on healthy tissue. Because of unique combinations of risk factors, the gene chain of command in the same type of cancer phenotype is most likely different from person to person.

In previous papers, we defined the genomic fabric [24-28] of a given biological process as the transcriptome associated with the most interconnected and stably expressed gene network responsible for that process. We reported that expression level, control and coordination of fabric genes depend on tissue, race/strain, sex and sex hormones, age and environment etc. and change during development, progression of a disease, and in response to various stimuli. The Genomic Fabric Paradigm (GFP) switches focus from the most frequently altered genes in numerous phenotypically similar individuals (biomarkers) to the most important genes for the individual patient.

Using GFP, its advanced analytical tools and a new transcriptomic measure "gene commanding height" we here propose a procedure to identify the command genes which we believe are the most legitimate targets for personalized anticancer therapy.

\section{Materials and Methods}

\subsection{Strategy}

Our strategy relies on collecting small pieces from histopathologically distinct regions of a tumor, split each piece in four and profile the transcriptome of each quarter. The splits (biological replicas) are like one system tuned to slightly different local conditions. The procedure is illustrated with a metastatic case of clear cell renal carcinoma.

Profiling four biological replicas provides enough accuracy for three independent measures of each gene: i) average expression level, ii) expression variability and iii) expression coordination with every other gene. Comparing the average expression levels of individual genes in a tumor cancer sample with respect to the adjacent normal tissue identifies the significantly regulated and turned on/off genes in that sample. Expression variability among biological replicas is an indirect estimate of the control exerted by the homeostatic mechanisms to stabilize the abundance of key transcripts within narrow interval regardless of environmental fluctuations. Most likely the commanded genes have the expression levels tied to the commander's. Therefore, the commanded genes are among the synergistically and antagonistically expressed partners of the commander and can be identified by the significant pair-wise Pearson correlation of their expression levels within biological replicas.

\subsection{Tumor Samples}

We have profiled the gene expression in frozen specimens from a 74y old man with metastatic clear cell renal cell carcinoma. The study was part of a project of Dr. DA Iacobas approved by New York Medical College's and Westchester Medical Center (WMC) Committees for Protection of Human Subjects, commonly known as Institutional Review Boards (IRBs) by L-11,376. The approval granted access to frozen cancer specimens from the WMC Pathology Archives and depersonalized pathology reports, waiving patient's informed consent.

Four samples were collected from each of NORmal cortex and two Primary Tumor regions A and B (PTA, PTB) of the right kidney and from a metastatic chest wall lesion (MET). PTA and PTB regions were selected based on their gross anatomical differences. The samples were small $\left(2-8 \mathrm{~mm}^{3}\right)$ and chosen to be as homogeneous as possible. However, cells of different phenotypes were not completely eliminated and expression of their genes might have affected the reported results.

\subsection{Microarray}

We used our standard protocol for extraction, reverse transcription, fluorescent labeling and hybridization with Agilent $4 \mathrm{x} 44 \mathrm{k}$ two color gene expression human microarrays of total RNA [29]. Raw and processed data complying with the Minimum Information About a Microarray Experiments (MIAME) have been deposited and are available at [30].

A gene was listed as differentially expressed between the regions $\alpha$ and $\beta$ if the absolute expression ratio $\mathrm{x}$ exceeded the unit by twice of the pooled expression variabilities of that gene in the two regions (Eq. 1)

$$
\left|x_{i}^{(\alpha, \beta)}\right|>1+\sqrt{2\left(\left(C V_{i}^{(\alpha)}\right)^{2}+\left(C V_{i}^{(\beta)}\right)^{2}\right)}
$$

where $\mathrm{CV}_{\mathrm{i}}^{(\alpha / \beta)}$ is the coefficient of variation in the indicated region.

The differential expression was considered as significant if the p-value of the heteroscedastic $t$-test computed with a Bonferroni type correction applied to the redundancy group of spots probing the same transcript [30] was less than 0.05. The method to identify the regulated genes was validated in many of our previous transcriptomic studies by comparing the microarray results with those obtained by qRT-PCR or Western blotting (e.g.: [17, 32, 33])

Expression control of a gene in a particular region was computed as the complement to $100 \%$ of the coefficient of variation of the normalized expression levels in the four biological replicas profiled in that region. 
Expression coordination of two genes $i$ and $j$ in a region $\alpha$ was determined by computing the pair-wise Pearson correlation coefficient $\rho_{\mathrm{ij}}{ }^{\alpha}$ between their corresponding $(\log 2)$ levels in the biological replicas of that region.

Gene Commanding Height (GCH) score of gene $i$ in region $\alpha$ was computed as:

$$
\begin{aligned}
& G C H_{i}^{(\alpha)}=\frac{\left\langle C V_{i}^{(\alpha)}\right\rangle_{\Gamma}}{C V_{i}^{(\alpha)}} \exp \left(\frac{4}{N-1} \sum_{j=1, j \neq i}^{N}\left(\rho_{i j}^{(\alpha)}\right)^{2}-1\right) \\
& \alpha=\text { NOR, PTA, PTB, MET }
\end{aligned}
$$

where $N$ is the number of all analyzed distinct genes.

\section{Results}

\subsection{Clear Cell Renal Cell Carcinoma CCRCC is Transcriptomically Heterogeneous}

Figure 1A presents the percentages of differentially expressed genes when NORmal, Primary Tumor A, Primary Tumor B and METastatic regions (NOR, PTA, PTB and MET) were compared to each other. With respect to NOR, $15.0 \%$ of the genes were significantly up- or down-regulated in PTA, $11.7 \%$ were regulated in PTB and 23.8\% in MET. Experimental data indicate that MET originated from PTA (only 3.6\% differentially expressed genes) rather than from PTB (23.8\% differentially expressed genes). Interestingly, more genes were differentially expressed between the two primary tumor regions (19.4\%) than between each tumor region and the reference tissue. The differences between PTA and PTB and similarities between PTA and MET are evident in Figure 1B, which presents the regulation of cancer biomarkers selected from [34].

\subsection{Cell Proliferation and Spread Increased in CCRCC to Counter Up-regulation of the Immune Response}

Phenotypic changes beyond cut-off limits trigger an immune attack to kill and eliminate aberrant cells. This is consistent with our finding that $19 \%$ of the 130 genes involved in the chemokine signaling [35] were regulated in PTA, $11 \%$ in PTB and $18 \%$ in MET. These percentages indicate also that the immune response was lower in PTB than in PTA and MET, concordant with all other findings of less severe CCRCC in PTB than PTA. However, although attacked by the immune system, cancer cells acquire proliferation rates that exceed tissue control mechanisms. Indeed, in all three cancer regions we found up-regulation of the cyclins CCNB1IP1, CCNC and CCND2 while none of the cyclin-dependent kinase inhibitors was affected. Cyclin-dependent kinases CDK13, CDK4, CDK5, CDK5R1 and CDK9 were up-regulated in both PTA and MET but not in PTB, perhaps explaining why the faster growing PTA cells metastasized to the chest wall.

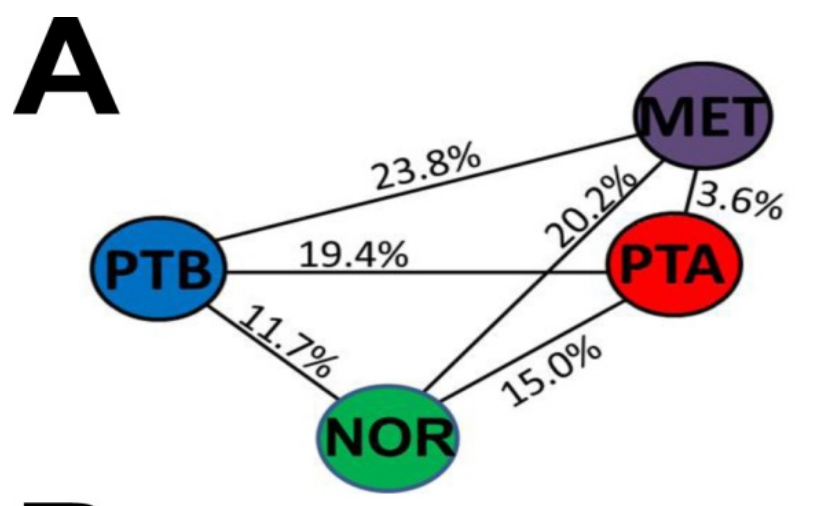

B

\begin{tabular}{|c|l|l|l|c|l|l|l|}
\hline GENE & PTA & PTB & MET & SYmbol & PTA & PTB & MET \\
\hline ABL1 & & & & JAK3 & & & \\
\hline AKT1 & 3.00 & & & KDR & & & \\
\hline APC & 2.02 & & 2.40 & KIT & & & \\
\hline ATM & & & & KRAS & & & 4.65 \\
\hline BRAF & 1.70 & & & LCP1 & & & \\
\hline CDH1 & & & & MET & & & \\
\hline CDKN2A & & & & MLH1 & & & \\
\hline CSF1R & & -2.12 & & MTOR & & & \\
\hline CTNNB1 & & & & NME1 & & & \\
\hline EGFR & & -2.55 & & NNMT & & & \\
\hline ERBB2 & & & & NOTCH1 & 1.69 & & 1.99 \\
\hline ERBB4 & & & & NPM1 & & & \\
\hline FBXW7 & 1.60 & & 2.01 & NRAS & 1.83 & & 2.42 \\
\hline FGFR1 & & & & PDGFRA & & 2.25 & \\
\hline FGFR2 & & & 2.41 & PIK3CA & & & \\
\hline FGFR3 & & & & PTEN & 2.50 & & 2.85 \\
\hline FLT3 & & & & PTPN11 & & & \\
\hline GNA11 & & & & RB1 & & & \\
\hline GNAQ & & & & RET & & & \\
\hline GNAS & & & & SMAD4 & & & \\
\hline HIF1A & & 1.99 & & SMARCB1 & & & 2.62 \\
\hline HNF1A & & & & SMO & & & \\
\hline HRAS & 1.88 & & 2.13 & SRC & 2.56 & & \\
\hline IDH1 & & & & STK11 & & & \\
\hline JAK2 & & & 2.41 & TP53 & & & \\
\hline JAK3 & & & & VHL & & & \\
\hline & & & & & & \\
\hline
\end{tabular}

Figure 1. (A) Percentages of differentially expressed genes when comparing the groups to each-other. Note the significantly lower percentage of differentially expressed genes between PTA and MET. (B). Regulation of some "known" cancer biomarkers. Red/green/blank square indicates whether that gene was significantly up-/down-/not regulated in the indicated region with respect to the control tissue. 
In addition to increased proliferation of cancer cells, cancer-inducing molecular factors can spread to neighboring cells via gap junction channels. We found that GJA4 gene encoding the vascular gap junction protein connexin 37 (Cx37) was up regulated by 2.1x in PTA, 2.0x in PTB and 2.4x in MET. GJB2, encoding Cx26, was 2.1x up-regulated in PTA, 1.8x down-regulated in PTB and 2.4x up-regulated in MET, while GJC1, encoding Cx45, was not regulated in PTA and MET but 2.6x down-regulated in PTB. Down-regulation of two important connexin genes in PTB suggests not only less synchronization of cancer development in the neighboring cells but also different tumorigenesis mechanisms than in PTA and MET regions.

\subsection{CCRCC Increased the Overall Expression Control and Coordination}

The overall expression variability among biological replicas was significantly reduced in all CCRCC groups with respect to NOR group indicating increased control of transcript abundances (Figure 2A). Analysis of expression correlation (principle presented in Figure 2B) also revealed substantial increases in expression coordination (both synergistic and antagonistic) in all CCRCC groups with respect to NOR samples (Figure 2C). Note that both expression control and expression coordination had smaller increases in the region also exhibiting the least expression regulation (PTB).

\subsection{CCRCC Rearranged the Gene Networks}

Expression coordination of two genes indicates whether they are inter-connected in a gene network. We found that all pathways analyzed so far (apoptosis, chemokine signaling, mTOR signaling, VEGF signaling, oncogenes and mitochondrial genes) as well as their interplays exhibited significant network rearrangement. Figure 3 illustrates the expression inter-coordination for a group of 20 mitochondrial ATP-synthases. CCRCC not only increased the overall expression coordination of these genes, but it also modified their partnerships, indicating changes in mitochondrial function. For instance, ATP6 (mitochondrially encoded ATP synthase 6) has no significant coordination partner in NOR but it is independently expressed with ATP5G2. However, it has 4 synergistically expressed partners in PTA including ATP5G2, 1 in PTB and 11 in MET.

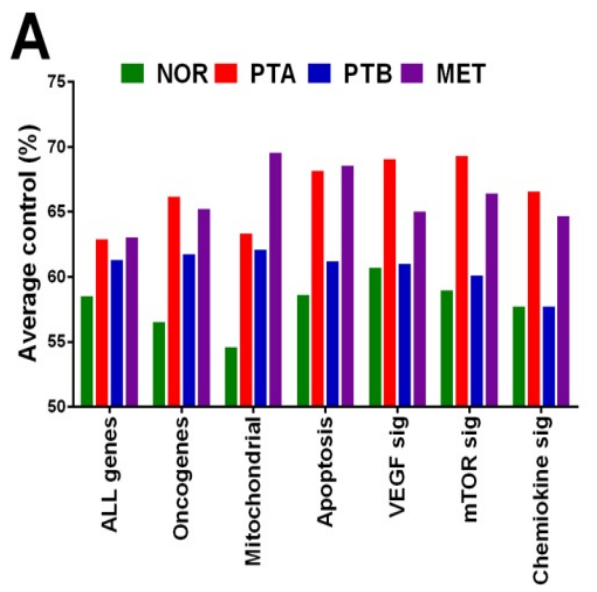

B

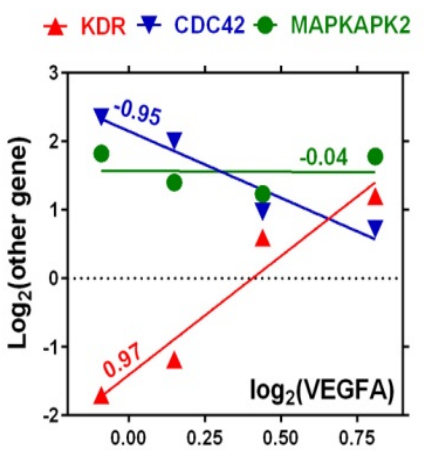

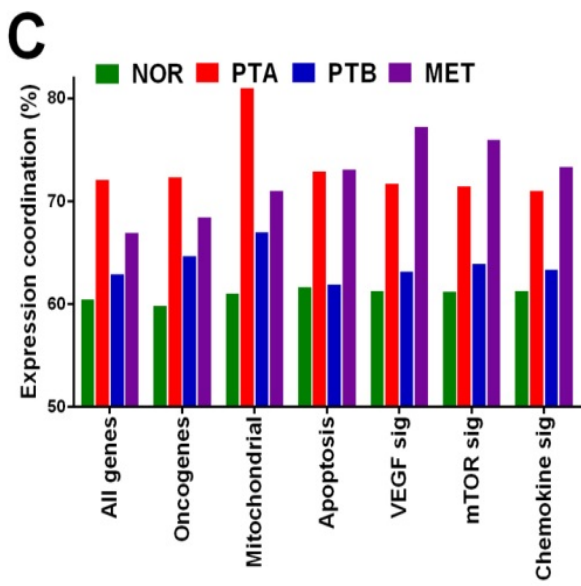

Figure 2. CCRCC increases expression control and coordination. (A). Average control of gene expression level among biological replicas of the four regions. The overall reduction of the coefficient of variation (CV) was evident for all genes as well as for the separately analyzed pathways. Note that $\mathrm{CV}$ reduction was smaller for PTB samples than for PTA and MET ones. (B). Examples of genes that are synergistically $(K D R=$ kinase insert domain receptor (a type III receptor tyrosine kinase)), antagonistically $(C D C 42=$ cell division cycle 42$)$ and independently $(M A P K A P K 2=$ mitogen-activated protein kinase-activated protein kinase 2) expressed with VEGFA (transcript variant 1) in PTB. Numbers above linear regression lines are the Pearson pair-wise correlation coefficients between the sets of (log2) expression levels within biological replicas of the correlated genes. (C). Coordination percentages when all genes are considered and when the analysis is restricted to separately analyzed groups of genes/pathways. Note the increase of coordination percentage in cancer samples. Observe also that the mitochondrial genes were the most affected. 

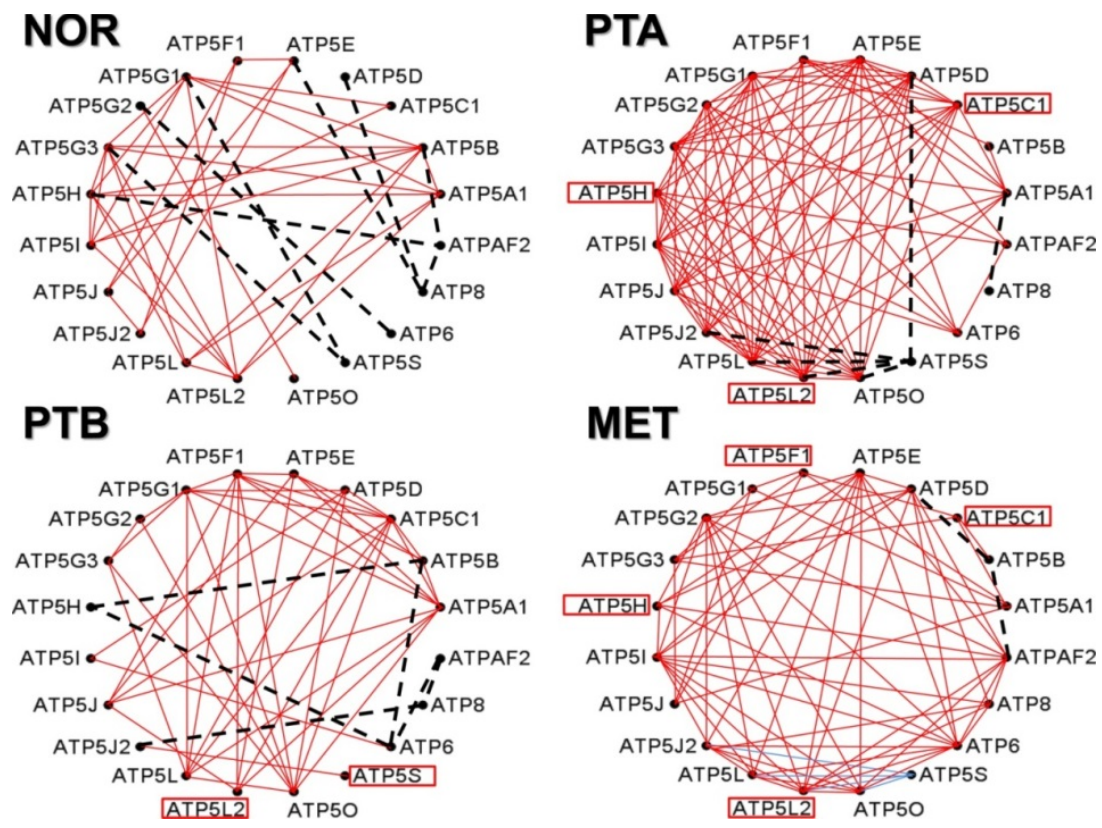

Figure 3. Expression coordination among 20 mitochondrial ATP-synthases in the four profiled regions. A red/blue/(dashed) black line indicates that the linked genes are $(\mathrm{p}<0.05)$ synergistically, antagonistically or independently expressed, while a missing line means lack of statistical evidence to characterize the coordination. Percentages of coordination for these genes were respectively: 64\% (NOR), 82\% (PTA), 70\% (PTB) and 78\% (MET). Note the increase of coordination in cancer samples and the changes in expression pairing. Gene symbol in a square box (e.g., ATP5L2) indicates significant up-regulation in that region with respect to the normal kidney tissue.

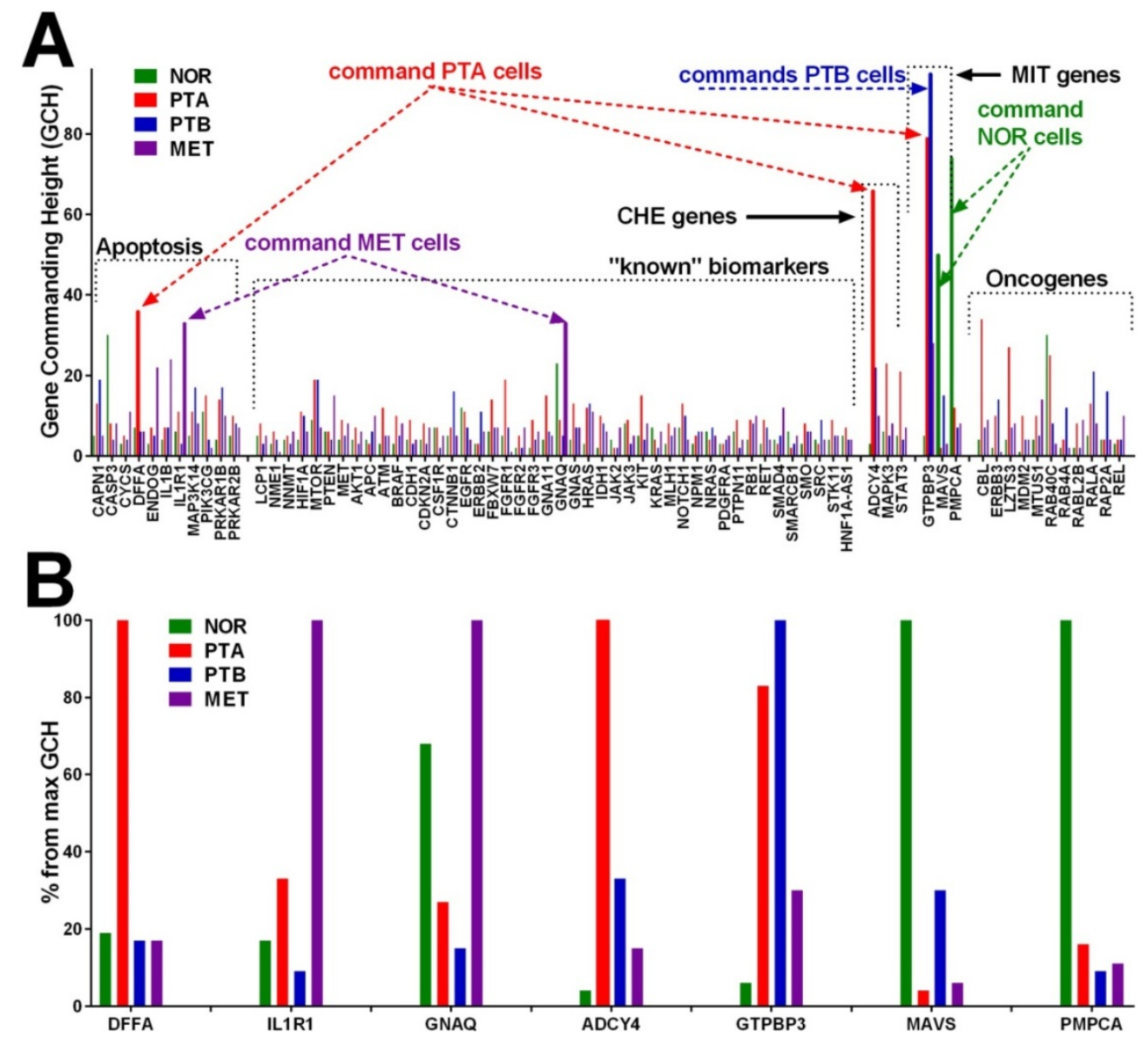

Figure 4. Gene Commanding Height. (A) GCH of several "known” cancer biomarkers, oncogenes, mitochondrial genes (MIT) and genes involved in apoptosis and chemokine signaling (CHE). Color arrows indicate the command genes in each region. (B) Relative GCH of selected command genes in regions other than they command. 


\subsection{Histopathologically Distinct Regions have Different Command Genes}

We defined (Eq.2) the Gene Commanding Height (GCH) in a particular region as a composite score accounting for gene expression stability and pooled expression coordination with expressions of other genes within biological replicas of that region. GCH replaces our Gene Prominence Score [24-27] used to select the most important pathway genes. Figure 4A presents the commanding heights of several "known" cancer biomarkers, oncogenes, mitochondrial genes (MIT) and genes involved in apoptosis and chemokine signaling (CHE). Results show that the four regions have different gene hierarchies. Interestingly, the mitochondrial GTPBP3 (GTP binding protein 3) tops both PTA $(\mathrm{GCH}=79)$ and PTB (GPS = 95), while PMPCA (peptidase (mitochondrial processing) alpha, GPS $=74$ ) tops the normal tissue. Genes in MET were less clearly differentiated, the top gene, GNAQ (guanine nucleotide binding protein ( $\mathrm{G}$ protein), q polypeptide) GPS $=33$, being much less prominent than the command genes in other regions. Interestingly, GNAQ was the single biomarkers that exhibited a higher GCH in a cancer region.

Importantly, the commanding genes in one region can be commanded in other regions. Thus, manipulation of such a gene is expected to have profoundly different effects on histopathologically distinct regions of the tumor. Figure 4B presents the percentage from the maximum commanding heights of some command genes in other regions than they command. For this particular patient, GTPBP3 is $15 \mathrm{x}$ more powerful in PTA, 18x in PTB and 5x in MET cells than in NOR. In contrast, PMPCA is $6 x$ less powerful in PTA, $11 x$ in $\mathrm{PTB}$ and $9 \mathrm{x}$ in MET than in NOR region. Therefore, the most legitimate gene to target for this patient is GTPBP3.

\section{Discussion}

This study does not propose new universal biomarkers for a particular form of cancer in ALL humans. Since any cancer phenotype is the result of all gene alterations (albeit with uneven contributions), and that the number of possible combinations of gene alterations is practically infinite, we will never be able to determine the predictive value of all combinations in the meta-analysis of a really stratified population. Besides, the biomarkers are selected from the most frequently altered genes in cancer patients (indicating less effort of cell homeostasis to protect their sequence/expression as for low plyers) and therefore targeting them may not bring much therapeutic benefits. Instead, we propose a personalized procedure to identify for EACH patient the genes whose manipulation might have the largest impact on cancer cells because of their regulating the expression of numerous other genes while being highly protected by homeostatic mechanisms.

We chose the transcriptomic features because in the end almost all kind of mutations affects the transcription level of the affected gene. GFP characterizes the organizational principles [36] of the (here) kidney cortex transcriptome for each individual aiming to determine the critical limits of their alteration beyond which there is a spontaneous progression to CCRCC. GFP can use transcriptomic data obtained with any high throughput gene expression platform when at least four biological replicas are profiled from each condition.

The 4-replicas design provides adequate accuracy for the expression variability and expression control, two additional independent measures complementing the average expression level.

The expression variability can be used to estimate the strength of the homeostatic control of transcript abundance [35]. We have speculated that expression of genes that are critical for cell survival, differentiation and integration in a multicellular structure should be under a stricter control. Thus, identification of the most controlled genes in a particular condition will indicate the cell "priorities" in that condition. Until a point of no return, the cell is expected to increase the overall control of transcript abundances to attenuate/limit the damaging effects of a disease or an environmental stress. However, cells should have genes whose relaxed control allows easy adaptation to environmental fluctuations in each condition and for each functional pathway. Interestingly, in many other microarray studies [31, 33, 37-40] we observed an overall reduction of expression variability (indicating increased) control in tissues from diseased, genetically manipulated or stressed animals compared to their healthy counterparts. In this study, we found an overall increase of the expression control in all cancer regions, the smallest increase being observed in the region (PTB) having also a smaller number of regulated genes. Therefore, we believe that increase of the expression control can be a universal criterion to assess the cancer stage.

The expression correlation indicates what genes can be included in functional pathways as satisfying a kind of "transcriptomic stoichiometry" [38]. When two genes are synergistically expressed the synthesis rates of the encoded proteins oscillate in synchrony while these rates manifest opposite tendencies when the genes are antagonistically expressed. Expression synergism and antagonism are essential for the performance of functional pathways. We consider the synergistically and antagonistically expressed genes as interconnected in transcriptomic networks. Transcriptomic networks may cross cell borders via intercellular communication. Thus, regulation of certain genes in one cell has consequences on the expression level of other genes in neighboring cells, even when neighbors are of different types [26, 41].

If two genes are independently expressed then there is little chance that their encoded proteins are part of the same functional pathway. In this study, we found an overall increase of expression coordination in all cancer regions. And, again, the smallest increase was in the least affected region (PTB). We have also reported increase of expression coordination in previous microarray studies on mice 
subjected to chronic intermittent or constant hypoxia [40]. Therefore, we believe that increases in expression coordination can be also a universal criterion to assess the stage of a cancer.

While biomarkers are selected by the Principal Component Analysis [23] as the most informative subset of genes for the transcriptomic alterations, GFP uses the Prominence Gene Analysis [24-26] and the Gene Commanding Height [28] to establish the gene chain of command in each condition. Top commander genes are under stricter protection of the homeostatic mechanisms (and by consequence among the least alterable) while coordinating expression of most other genes of the pathway.

This study verified that in a heterogeneous tumor each histopathologically distinct region has a different set of commander genes. If a commander gene in cancer cells (like GTPBP3 here) has significantly lower GCH in normal tissue, then it could be a potential target to push cell physiological parameters beyond viable levels in cancer cells leading to selective destruction whilst preserving function/survival of cells in normal tissue.

It is possible that certain candidate genes have already FDA approved targeting therapies. Thus, our procedure could be a firm step towards a really personalized genomic cancer medicine.

In order to test the usefulness of our procedure we should overexpress the commanders that stimulate cancer cell death and/or tumor suppression or silence the commanders involved in proliferation and/or spreading. Unfortunately, we had no possibility to validate for this patient what manipulation of command genes can do. However, experiments on several mouse and human cancer cell lines are underway to test whether manipulation of command genes affects selectively the cells they command. Further studies on various cancer types are also necessary to test the usefulness of the overall expression control and expression coordination for cancer transcriptomic grading.

\section{Conclusions}

Instead of using standard gene panels with disputable predictive value for the current patient, we would likely profile the cancer nodules, identify the commander genes and target them.

\section{Acknowledgements}

Surgery was performed by Dr. R.J. Lafaro at Westchester Medical Center and the pathology report was written by Dr. M Zhong. Drs. Randy F. Stout (NYIT), Henry P. Godfrey (NYMC) and David C Spray (Einstein) are acknowledged for their valuable critical comments on the manuscript.

Funding: NYMC Department of Pathology

Author Contributions: SI conducted the microarray experiment and DAI developed the method, design the experiment and performed the analyses. Both authors contributed to the manuscript writing.

Competing Interests: no competing interests.

\section{REFERENCES}

[1] http://www.cancer.gov

[2] http://cancergenome.nih.gov

[3] https://tcga-data.nci.nih.gov/tcga

[4] Tomasetti C, Li L, Vogelstein B. Stem cell divisions, somatic mutations, cancer etiology, and cancer prevention. Science. 24;355(6331):1330-1334, 2017

[5] Vogelstein B, Papadopoulos N, Velculescu VE, Zhou S, Diaz LA Jr, Kinzler KW. Cancer genome landscapes. Science. 339(6127):1546-58.5, 2013.

[6] http://www.cancer.ca/en/cancer-information/cancer-101/what -is-a-risk-factor/genetic-risk/sporadic-mutations/?region=on

[7] Bultman SJ. The microbiome and its potential as a cancer preventive intervention. Semin Oncol. 43(1):97-106, 2016.

[8] Bagrodia A, Cha EK, Sfakianos JP, Zabor EC, Bochner BH, Al-Ahmadie HA, Solit DB, Coleman JA; Collaborators (11). Genomic biomarkers for the prediction of stage and prognosis of upper tract urothelial carcinoma. J Urol. pii: S0022-5347(16)00033-1, 2016.

[9] Chen J, Gong TT, Wu QJ. Parity and gastric cancer risk: a systematic review and dose-response meta-analysis of prospective cohort studies. Sci Rep. 6:18766, 2016.

[10] Karim S. Assessment of radiation induced therapeutic effect and cytotoxicity in cancer patients based on transcriptomic profiling. Int. J Mol Sci. 17(2). pii: E250, 2016.

[11] Nq KL , Morais C, Bernard A, Saunders N, Samaratunga H, Gobe G, Wood S. A systematic review and meta-analysis of immunohistochemical biomarkers that differentiate chromophobe renal cell carcinoma from renal oncocytoma. J Clin Pathol. pii: jclinpath-2015-203585, 2016.

[12] Schubert M, Junker K, Heinzelmann J. Prognostic and predictive miRNA biomarkers in bladder, kidney and prostate cancer: Where do we stand in biomarker development? J Cancer Res Clin Oncol. 142(8):1673-95, 2016.

[13] Yao J , Li ZH, Li YX, Zhang R, Zhang DG, Xu ZL, Wang LS, Wang JY. Association between the $-607 \mathrm{C}>$ A polymorphism in interleukin-18 gene promoter with gastrointestinal cancer risk: a meta-analysis. Genet Mol Res. 14(4):16880-7, 2015.

[14] Lucarelli G, Rutigliano M, Sanguedolce F, Galleggiante V, Giglio A, Cagiano S, Bufo P, Maiorano E, Ribatti D, Ranieri E, Gigante M, Gesualdo L, Ferro M, de Cobelli O, Buonerba C, Di Lorenzo G, De Placido S, Palazzo S, Bettocchi C, Ditonno P, Battaglia M. Increased Expression of the Autocrine Motility Factor is Associated With Poor Prognosis in Patients With Clear Cell-Renal Cell Carcinoma. Medicine (Baltimore) 94(46):e2117, 2015.

[15] Mo R, Peng J, Xiao J, Ma J, Li W, Wang J, Ruan Y, Ma S, Hong Y, Wang C, Gao K, Fan J. High TXNDC5 expression 
predicts poor prognosis in renal cell carcinoma. Tumor Biol. 37(7):9797-806, 2016.

[16] Kohane IS, Hsing M, Kong SW. Taxonomizing, sizing, and overcoming the incidentalome. Genet Med 14, 399-404, 2012.

[17] Iacobas DA, Iacobas S, Urban-Maldonado M, Scemes E, Spray DC. Similar transcriptomic alterations in Cx43 knock-down and knock-out astrocytes. Cell Commun. Adhes 15, 195-206, 2008.

[18] Amaro A, Esposito AI, Gallina A, Nees M, Angelini G, Albini A, Pfeffer U. Validation of proposed prostate cancer biomarkers with gene expression data: a long road to travel. Cancer Metastasis Rev. 33(2-3):657-71. Review, 2014.

[19] Klein EA , Cooperberg MR, Magi-Galluzzi C, Simko JP, Falzarano SM, Maddala T, Chan JM, Li J, Cowan JE, Tsiatis AC, Cherbavaz DB, Pelham RJ, Tenggara-Hunter I, Baehner FL, Knezevic D, Febbo PG, Shak S, Kattan MW, Lee M, Carroll PR. A 17-gene assay to predict prostate cancer aggressiveness in the context of Gleason grade heterogeneity, tumor multifocality, and biopsy undersampling. Europ. Urol 66, 550-560, 2014.

[20] Luo Y, Zhu X, Zhang P, Shen Q, Wang Z, Wen X, Wang L, Gao J, Dong J, Yang C, Wu T, Zhu Z, Tian Y. The clinical performance evaluation of novel protein chips for eleven biomarkers detection and the diagnostic model study. Int J Clin Exp Med. 8(11):20413-23, 2015.

[21] Wu CL, Schroeder BE, Ma XJ, Cutie CJ, Wu S, Salunga R, Zhang Y, Kattan MW, Schnabel CA, Erlander MG, McDougal WS. Development and validation of a 32-gene prognostic index for prostate cancer progression. PNAS 110 (15): 6121-6, 2013.

[22] Margolin G, Petrykowska HM, Jameel N, Bell DW, Young AC, Elnitski L. Robust detection of DNA hypermethylation of ZNF154 as a pan-cancer locus with in silico modeling for blood-based diagnostic development. J Mol Diagn. 18(2):283-98, 2016.

[23] Draghici S. Data analysis tools for DNA microarrays, Chapman \& Hall, Boca Raton, pp. 223-224, 2003.

[24] Iacobas DA, Iacobas S, Haddad GG. Heart rhythm genomic fabric in hypoxia. Biochem. Biophys. Res. Commun. 391(4):1769-1774, 2010.

[25] Iacobas DA, Iacobas S, Thomas N, Spray DC. Sex-dependent gene regulatory networks of the heart rhythm. Funct Integr Genomics. 10(1):73-86, 2010.

[26] Iacobas S, Iacobas DA. Astrocyte proximity modulates the myelination gene fabric of oligodendrocytes. Neuron Glia Biology 6(3): 157-169, 2010.

[27] Iacobas DA, Iacobas S, Chachua T, Goletiani C, Sidyelyeva G, Velíšková J, Velíšek L. Prenatal corticosteroids modify glutamatergic and GABAergic synapse genomic fabric: Insights from a novel animal model of infantile spasms. J Neuroendocrinol. 25, 964-979, 2013.

[28] Iacobas DA. The Genomic Fabric Perspective on the Transcriptome between Universal Quantifiers and Personalized Genomic Medicine. Biological Theory. 11(3): 123-137, 2016.

[29] Lee PR, Cohen JE, Iacobas DA, Iacobas S, Fields RD. Gene-regulatory networks activated by pattern-specific generation of action potentials in dorsal root ganglia neurons. Scientific Reports. 7:43765, 2017.

[30] http://www.ncbi.nlm.nih.gov/geo/query/acc.cgi?acc=GSE723 04

[31] Iacobas DA, Iacobas S, Urban-Maldonado M, Spray DC. Sensitivity of the brain transcriptome to connexin ablation, Biochimica et Biofisica Acta. 1711: 183-196, 2005. Review./

[32] Fan C, Iacobas DA, Zhou D, Chen Q, Gavrialov O, Haddad GG. Gene expression and phenotypic characterization of mouse heart after chronic constant and intermittent hypoxia. Physiol Genomics. 22: 292-307, 2005.

[33] Iacobas DA, Iacobas S, Li WE, Zoidl G, Dermietzel R, Spray DC. Genes controlling multiple functional pathways are transcriptionally regulated in connexin 43 null mouse heart. Physiol Genomics 20: 211-223, 2005.

[34] http://www.illumina.com/products/truseq_amplicon_cancer_ panel.html

[35] http://www.genome.jp/kegg-bin/show_pathway?hsa04062

[36] Spray DC, Iacobas DA. Organizational principles of the connexin-related brain transcriptome. J Membr Biol. 218(1-3):39-47, 2007.

[37] Iacobas DA, Fan C, Iacobas S, Spray DC, Haddad GG. Transcriptomic changes in developing kidney exposed to chronic hypoxia. Biochem Biophys Res Comm. 349(1), 329-338, 2006.

[38] Iacobas DA, Iacobas S, Spray DC Connexin43 and the brain transcriptome of the newborn mice. Genomics. 89(1), 113-123, 2007.

[39] Iacobas DA, Iacobas S, Spray DC. Connexin-dependent transcellular transcriptomic networks in mouse brain. Prog Biophys Mol Biol. 94(1-2):168-184, 2007. Review.

[40] Iacobas S, Iacobas DA. Effects of Chronic Intermittent Hypoxia on Cardiac Rhythm Transcriptomic Networks. In: Intermittent Hypoxia and Human Diseases, (Editors: L. XI, T.V. Serebrovskaya), New York: Springer. Pp. 15-28, 2012.

[41] Iacobas S, Thomas NM, Iacobas DA. Plasticity of the myelination genomic fabric. Mol Gen Genom. 287, 237-246, 2012. 